\title{
A RFID Explicit Tag Estimation Scheme for Dynamic Framed-Slot ALOHA Anti-Collision
}

\author{
Prapassara Pupunwiwat, Bela Stantic \\ Institute for Integrated and Intelligent Systems \\ Griffith University, Queensland, Australia \\ \{P.Pupunwiwat, B.Stantic\}egriffith.edu.au
}

\begin{abstract}
Radio Frequency Identification (RFID) systems consist of fast capturing radio frequency tags and networked electromagnetic readers. Despite the advances of the RFID technology, tag collision is a major problem and can be solved by using the anti-collision scheme. The current ALOHA-based approach suffers from tag starvation problem due to the inaccurate frame size. In this paper, we propose a "RFID Explicit Tag Estimation Scheme" (RETES) for a Dynamic Framed-Slot ALOHA (DFSA), which estimate precise number of tags around the reader and compatible with Electronic Product Code (EPC) Class 1 Generation 2. The preliminary findings indicated that our method achieves better outcome than existing techniques. We identified that the variations of specific RETES's parameters impact the DFSA's performance. Therefore, to achieve the best performance the parameters should be dynamically adjusted over the process of identification.
\end{abstract}

\section{INTRODUCTION}

RFID technology has gained significant momentum in the past few years. The core RFID technology can be traced back to World War II where it was used to distinguish between friendly and enemy aircrafts. Currently RFID technology is used in different systems such as: transportation, consumer packaging, security and access control, library system, and defence and military.

When numerous tags are present in the interrogation zone at the same time, the reader requires an ability to read data from individual tags. A technical scheme that handles tag collision without any interference is called an anti-collision protocol. The main focus of an anti-collision scheme is to read multiple tags as fast and reliably as possible. Two types of tag anti-collision algorithms widely used in RFID systems are the Tree-based deterministic anti-collision and the ALOHA-based probabilistic anti-collision.

In this study, we propose a RFID Explicit Tag Estimation Scheme (RETES) for Backlog estimation and framesize estimation, which is compatible with Dynamic FramedSlot ALOHA (DFSA). The aim of RETES is to identify the best variables that can predict the most accurate number of tags within an interrogation zone, using information based on collision slots and empty slots. Accurate estimation of number of tags leads to accurate frame-size estimation and system efficiency. Initial findings have indicated that RETES with lower values for the parameters have a better impact on the performances of DFSA. The initial $Q$ values and number of tags within the reader zone also influence the performance of RETES.
The remainder of this paper is organised as follows: In section 2 we provide background information related to RFID tag collisions and anti-collision schemes, and discussion of the Dynamic Framed-Slot ALOHA and its limitations. In section 3 we present a core of new methodology, the RFID Explicit Tag Estimation Scheme. In section 4, we present our experimental evaluation, results, and analysis. We conclude our paper in section 5 .

\section{RFID BACKGROUND}

RFID technology is a contactless identification technology that identifies electronic tags attached to items. There are several methods of identification but the most common is to store a serial number that identifies a person or object such as Electronic Product Code (EPC).

Simultaneous transmissions in RFID systems lead to collisions as the readers and tags typically operate on the same channel. The tag collision in RFID systems, also known as Multi-Access, happens when multiple tags are energised by the RFID tag reader simultaneously, and reflect their respective signals back to the reader at the same time.

The various types of tag anti-collision approaches for tag collision can be reduced to two basic types: Tree-based deterministic approach and ALOHA-based probabilistic approach. The Tree-based approach is slow and introduces identification delay but leads to fewer collisions. In contrast, tags respond at randomly generated times in an ALOHA-based approach. If a collision occurs, colliding tags will have to identify themselves again after waiting a random period of time [1]. This technique is faster than Tree-based but suffers from tag starvation problem where not all tags can be identified due to the random nature of chosen time.

\section{A. Dynamic Framed-Slot ALOHA Algorithm}

In DFSA, each tag in an interrogation zone selects one of the given $N$ slots to transmit its identifier; and all tags will be recognised after a few frames. Each frame is formed of specific number of slots that is used for the communication between the readers and the tags. The frame-size is dynamically changed according to estimated number of Backlog, which is a number of tags that have not been read. There have been several researches to improve the accuracy of frame-size by implementing a Frame Estimation Tool [2], [3], [4]. 
According to the DFSA protocol, the reader picks tag within an interrogation zone by the command "Select"; then issues "Query", which contains a 'Q' parameter to specify the framesize (frame-size $\mathrm{F}=2^{Q}-1$ ). Each selected tag will pick a random number between 0 to $2^{Q}-1$ and put it into its slot counter. The tag, which picks zero as its slot number, will respond and backscatter its EPC to reader. Then, reader issues "QueryRep" or "QueryAdjust" command to initiate another slot [5]. In ALOHA-based anti-collision, there are three kinds of slot as shown in Figure 1: 1) Successful slot where there is only one tag reply; 2) Empty slot where there is no tag reply; and 3) Collision slot where there is more than one tag reply.

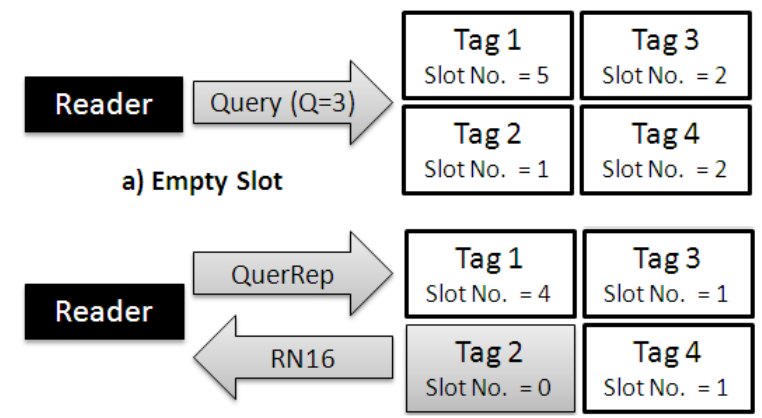

b) Successful Slot

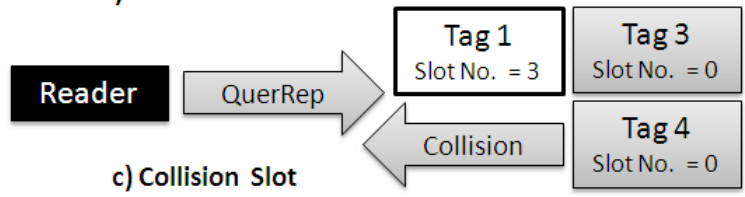

Fig. 1. Empty Slot, Successful Slot, and Collision Slot in EPC Class 1 Gen2 Protocol

\section{B. Backlog Estimation Schemes}

There are different Backlog estimation methods proposed in the literature [6], [7], [8], [9], [10]. These methods predicted number of tags, which may occur for the next identification round, by using information from collision slots and/or empty slots of the current frame. Major methods are explained as follows:

1) Schoute backlog estimation technique: Schoute developed a Backlog estimation technique for Dynamic FramedSlot ALOHA using Poisson distributed [6]. The Backlog after the current frame $\mathrm{Bt}$ is given by equation:

$$
B t=2.39 * c
$$

where c represents the number of collided slot in the current frame. This technique has the best performance, where fewest frames were used compared to other algorithms.

2) Lowerbound backlog estimation technique: The estimation function is obtained under the assumption that a collision involves at least two different tags. Therefore, Backlog after the current frame $\mathrm{Bt}$ is given by equation:

$$
B t=2 * c
$$

where $\mathrm{c}$ is the number of collided slot in the current frame.
3) Other backlog estimation techniques: There have been several researches on Backlog estimation including C-Ratio method [7], Chen1 and Chen2 methods [8], Vogt method [9], and Bayesian method [10]. These methods are either having worse performances than simple Schoute's method or too complicated to implement for RFID system. Therefore, we only compare our method to Schoute and Lowerbound methods, since the two methods are simple and have excellent performances.

\section{RETES METHODOLOGY}

In order to overcome shortcomings of existing methods, we propose a RFID Explicit Tag Estimation Scheme (RETES) to estimate accurate number of tags and frame-size for DFSA protocols using collision slots and empty slots prediction. Accurate estimation of number of tags will lead to accurate frame-size estimation for the next round of identification process, which then results in a minimal number of slots and frames issued by a reader. This section will describe the newly proposed RETES; the specific requirements for tag estimation; initial $Q$ value; and suggest frame-size.

\section{A. RFID Explicit Tag Estimation Scheme - RETES}

RETES uses a number of parameters to predict collision slots and empty slots for new identification round. RETES method aims to obtain the optimal parameters in order to calculate and predict the closest number of remaining tags for the upcoming round of identification. We assume that for the current identification round, each collision slot has at least two tags that collided. However, we cannot know for sure how many tags actually caused the collision. Because there are exactly one tag per successful slot, we do not take successful slots into consideration. On the other hand, we assume that the empty slots will continuously occur during the next rounds of identification, regardless of the frame-size. Thus, RETES method is introduced to find the optimal parameters and to predict the number of remaining tags for upcoming round, using information from both collision slots and empty slots of the current frame.

RETES uses parameter 2.0 to predict the number of $\mathrm{col}$ lision slots after the first round of identification. Parameter 2.0 is chosen according to the assumption that at least two tags collided per collision slot. Since the number of tags is supposedly unknown at the beginning of the identification, a simple frame-size prediction using variable 2.0 is chosen for the next $Q$ adjust. Equation (1) shows Backlog estimation using parameter 2.0 for collision slots prediction.

$$
\text { Backlog }=\operatorname{Round}(2.0 * c)
$$

where $c$ is the number of collided slot in the current frame.

After the second round of identification, the RETES uses different parameter between 2.0 and 2.5 to predict the number of collision slots. Since a collision slot engages at least two tags, we assume that the parameter for collision slots calculation falls between 2.0 and 2.5 (more than 2 but possibly less than 2.5). However, number of tags per collision slot can 
be more than 2.5 tags. According to Schoute's method [6], which has the best Backlog estimation, the parameter used is 2.39. Therefore, we assume that the optimal parameter falls between 2 and 2.5. Variables between 0.1 and 0.5 are also used to predict the number of empty slots for the upcoming round. Since an empty slot does not engage any tag, we assume that the parameter for empty slots calculation falls between 0.1 and 0.5. Equation (2) shows Backlog estimation using variable $\mathrm{V}_{1}$ for collision slots prediction and variable $\mathrm{V}_{2}$ for empty slots prediction.

$$
\text { Backlog }=\operatorname{Round}\left(V_{1} * c+V_{2} * e\right)
$$

where $c$ is collision slot; $e$ is empty slot; $V_{1}$ is variable between 2.0 and 2.5; and $\mathrm{V}_{2}$ is variable between 0.1 and 0.5 with increments of 0.1 .

\section{B. Slot Observation and Initial $Q$-Value}

According to DFSA algorithm, the reader picks tag within an interrogation zone by the command "Select"; then issues "Query", which contains a 'Q' parameter to specify the framesize, $\left[\mathrm{F}=2^{Q}-1\right]$. For our methodology, initial $Q$ value can be any number between 1 and 10 since we assume that a reader can at most pick up no more than 800 tags per round. After the first round of identification, collision slots and empty slots will be observed and used, to estimate number of tags. After the number of tags has been estimated, frame-size for the next identification round can be configured. The suggested framesize is explained in the following sub-section.

\section{Suggested Frame-Size}

The suggested frame-size for our methodology is set according to estimated number of tags. For example, if estimated number of tags is around 100 tags, the suggested frame-size would have a $Q$ value of 7 . Since the frame-size is calculated by $2^{Q}-1$, the frame-size where $\mathrm{Q}=7$ will allow at most 128 tags $\left(0\right.$ to $\left.2^{7}-1\right)$ to be identified. Therefore, if the estimated number of tags is between 65 and 128 tags, the suggested $Q$ would equal to 7. Table I shows Minimum and Maximum number of tags allowed per suggested frame-size.

TABLE I

SugGeSTED FrAME-SIZE FOR SPECIFIC ESTIMATED NUMBER OF TAGS. MAXIMUM TAGS OF EACH FRAME-SIZE IS CALCULATED BY $2^{Q}-1$

\begin{tabular}{|c|c|c|c|c|c|}
\hline $\mathbf{2}^{Q}$ & $\mathbf{2}^{1}$ & $\mathbf{2}^{2}$ & $\mathbf{2}^{3}$ & $\mathbf{2}^{4}$ & $\mathbf{2}^{5}$ \\
\hline Min & 0 & 3 & 5 & 9 & 17 \\
\hline Max & 2 & 4 & 8 & 16 & 32 \\
\hline \multicolumn{7}{|l|}{} \\
\hline $\mathbf{2}^{Q}$ & $\mathbf{2}^{6}$ & $\mathbf{2}^{7}$ & $\mathbf{2}^{8}$ & $\mathbf{2}^{9}$ & $\mathbf{2}^{10}$ \\
\hline Min & 33 & 65 & 129 & 257 & 513 \\
\hline Max & 64 & 128 & 256 & 512 & 1024 \\
\hline
\end{tabular}

\section{EXPERIMENTAL EVALUATION}

In order to show the significance of our method, we conducted two experimental evaluations and compared our method to the existing techniques. In this section, we describe the data sets used in each experiment; present the results of the experiment; and provide an analysis upon these results.

\section{A. Data Sets}

Different tag sets are simulated in the experiment, we tested performance of RETES method versus Schoute (Sch) and Lowerbound (LB) method. The data used for the experiment has been randomly generated to reflect a real world scenario.

The aims of the experiment are to find the impact of different tag sets; to find the impact of different initial $Q$; and to find optimal parameters that produce the minimal number of slots and frames.

- There are three tag sets for different tag sets comparison comprising of 200, 250, and 300 tags. The initial $Q$ of for each tag set is fixed to 8 .

- A tag set utilised for different initial $Q$ comparison comprising 200 tags. Different initial $Q$ of 6, 7, and 8 are applied on the tag set.

\section{B. Results}

Results from the experiment show that different parameters impact performances of RETES. Figure 2 and Table II show that different number of tags within the reader's range resulted in different total number of slots and frames queried by RETES, Schoute (Sch), and Lowerbound (LB) methods. Thus, we compare RETES's performance based on both number of slots and frames. Sch and LB methods have a constant parameter of 2.39 and 2.0 respectively for number of collision slots prediction.

Figure 2 demonstrates that there are several major variables used by RETES which ensure good performances, compared with Sch and LB methods. These parameters are $2.0<=\mathrm{V}_{1}$ $<=2.2$ and $0.1<=\mathrm{V}_{2}<=0.2$. Considering different tag sets, it can be seen that when there are lower number of tags, especially 200 and 250 tags, RETES performs better or is in-line with Sch and LB methods using optimal parameters. However, when number of tags exceed the capability of number of available slots $\left(Q^{8}=256\right.$ slots $)$, specifically for 300 tags, the performances of RETES using optimal parameters degenerated.

TABLE II

NUMBER OF SLOTS AND FRAMES COMPARISON USING DIFFERENT TAG SETS AND INITIAL Q; RETES VERSUS SCH AND LB

\begin{tabular}{|c|c|c|c|c|c|c|}
\hline & \multicolumn{3}{|c|}{ Different tag sets } & \multicolumn{3}{c|}{ Different Initial Q } \\
\cline { 2 - 7 } & \multicolumn{2}{|c|}{ No. Slots/No. Frames } & \multicolumn{3}{c|}{ No. Slots/No. Frames } \\
\cline { 2 - 7 } & $\mathbf{2 0 0}$ & $\mathbf{2 5 0}$ & $\mathbf{3 0 0}$ & $\mathbf{Q ~ 6}$ & $\mathbf{Q ~ 7}$ & $\mathbf{Q ~ 8}$ \\
\hline $\mathbf{2 . 0 , 0 . 1}$ & $564 / 7$ & $654 / 9$ & $860 / 9$ & $616 / 10$ & $558 / 9$ & $564 / 7$ \\
$\mathbf{2 . 0 , 0 . 2}$ & $552 / 5$ & $728 / 7$ & $864 / 9$ & $656 / 7$ & $568 / 7$ & $552 / 5$ \\
$\mathbf{2 . 0} \mathbf{0 . 3}$ & $576 / 4$ & $728 / 7$ & $928 / 7$ & $672 / 6$ & $608 / 6$ & $576 / 4$ \\
$\mathbf{2 . 1 , 0 . 1}$ & $564 / 7$ & $656 / 8$ & $858 / 8$ & $626 / 7$ & $568 / 7$ & $564 / 7$ \\
$\mathbf{2 . 1} \mathbf{0 . 2}$ & $552 / 5$ & $728 / 7$ & $872 / 7$ & $656 / 6$ & $568 / 7$ & $552 / 5$ \\
$\mathbf{2 . 1} \mathbf{0 . 3}$ & $576 / 4$ & $728 / 7$ & $880 / 7$ & $672 / 6$ & $608 / 6$ & $576 / 4$ \\
$\mathbf{2 . 2 , 0 . 1}$ & $552 / 5$ & $728 / 7$ & $872 / 7$ & $656 / 6$ & $568 / 7$ & $552 / 5$ \\
$\mathbf{2 . 2 , 0 . 2}$ & $552 / 5$ & $728 / 7$ & $880 / 7$ & $656 / 6$ & $568 / 7$ & $552 / 5$ \\
$\mathbf{2 . 2 , 0 . 3}$ & $576 / 4$ & $728 / 7$ & $880 / 7$ & $672 / 6$ & $608 / 6$ & $576 / 4$ \\
\hline LB & $562 / 8$ & $654 / 10$ & $860 / 9$ & $606 / 11$ & $556 / 10$ & $562 / 8$ \\
\hline Sch & $552 / 5$ & $700 / 8$ & $810 / 8$ & $626 / 7$ & $600 / 7$ & $552 / 5$ \\
\hline
\end{tabular}

Figure 2 also demonstrates that different initial $Q$ influence the performance of RETES. Looking at low initial $Q$ and high number of tags from Figure 2 and Table II, particularly where 
RETES versus Sch \& LB

Number of slots comparison

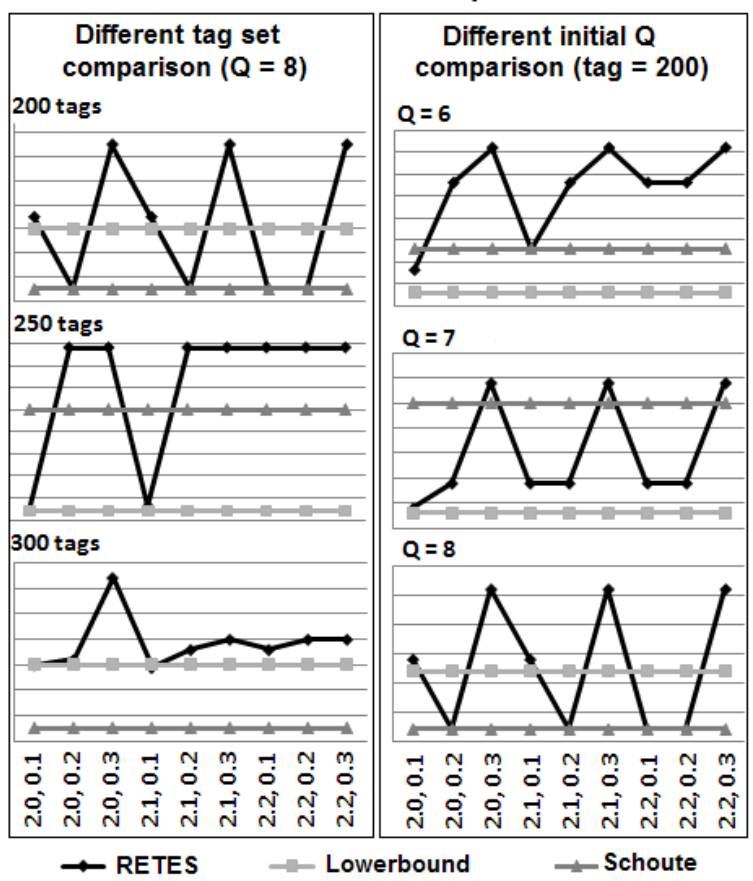

Fig. 2. Number of slots comparison of RETES method versus Sch and LB methods using different tag sets and initial Q

$\mathrm{Q}=6$ and number of tags is 200 tags, performance of RETES is not good compared with both Sch and LB. However, when initial $Q$ of 7 and 8 are used while number of tags stay 200, performance of RETES improved, considering only optimal parameters $2.0<=\mathrm{V}_{1}<=2.2$ and $0.1<=\mathrm{V}_{2}<=0.2$.

\section{Analysis}

Overall analysis of the experiment is presented in Table III. Performances of the RETES methods are classified into three categories: good, in-line, and poor. The table demonstrated that when using higher initial $Q$, RETES has good performance compared with Sch and LB methods. In contrast, when initial $Q$ is low, RETES's performance degenerated but still inline with existing methods. Table III shows that the RETES performed the best overall, this is the case when the optimal parameters $2.0<=\mathrm{V}_{1}<=2.2$ and $0.1<=\mathrm{V}_{2}<=0.2$ are considered.

TABLE III

IMPACT ANALYSIS OF INITIAL Q AND NUMBER OF TAGS ON PERFORMANCES OF RETES VERSUS SCH AND LB METHODS

\begin{tabular}{|c|c|c|c|c|c|c|c|}
\hline & \multicolumn{2}{|c|}{ Initial Q } & \multicolumn{2}{c|}{ No. of Tags } & \multicolumn{3}{c|}{ Performances } \\
\cline { 2 - 8 } & Low & High & Low & High & Good & Inline & Poor \\
\hline 200 tags & - & Y & Y & - & Y & - & - \\
250 tags & - & Y & Y & - & Y & - & - \\
300 tags & Y & - & - & Y & - & Y & - \\
\hline \multicolumn{8}{|l}{} \\
\hline Q6 (64) & Y & - & - & Y & - & Y & - \\
Q7 (128) & Y & - & - & Y & - & Y & - \\
Q8 (256) & - & Y & Y & - & Y & - & - \\
\hline
\end{tabular}

\section{CONClusion}

In this study, we have identified the significance of RFID tag anti-collision and developed an efficient method to minimise tag starvation problem. We have proposed a RFID Explicit Tag Estimation Scheme (RETES), which estimates the precise number of tags around the reader using existing information on collision slots and empty slots to predict the correct framesize and improve the system efficiency of the ALOHA-based DFSA.

In the experimental evaluations, the results and analysis of experiments have shown that the parameter $2.0<=\mathrm{V}_{1}<=$ 2.2 and $0.1<=\mathrm{V}_{2}<=0.2$ is the optimal parameter for RETES. The performance of the proposed method depends on the selected initial $Q$ and number of tags within the reader zone. RETES has a better performance when using higher initial $Q$.

From these results, we conclude that the empty slots and collision slots both have impact on the Backlog prediction. In addition, different initial $Q$ has an impact on the RETES's performance. Therefore, to achieve the best performance the parameters should be adaptively adjusted over the process of identification.

\section{ACKNOWLEDGMENT}

This research is partly supported by ARC (Australian Research Council) grant no DP0557303.

\section{REFERENCES}

[1] J. D. Shin, S. S. Yeo, T. H. Kim, and S. K. Kim, "Hybrid Tag Anticollision Algorithms in RFID Systems," in Computational Science ICCS 2007, vol. 4490. Springer Berlin/Heidelberg, 2007, pp. 693-700.

[2] S. R. Lee, S. D. Joo, and C. W. Lee, "An enhanced dynamic framed slotted aloha algorithm for rfid tag identification," in MOBIQUITOUS '05: Proceedings of the The Second Annual International Conference on Mobile and Ubiquitous Systems: Networking and Services. Washington, DC, USA: IEEE Computer Society, 2005, pp. 166-174.

[3] C. W. Lee, H. Cho, and S. W. Kim, "An Adaptive RFID Anti-Collision Algorithm Based on Dynamic Framed ALOHA," IEICE Transactions, vol. 91-B, no. 2, pp. 641-645, 2008.

[4] H. Cho, W. Lee, and Y. Baek, "LDFSA: A Learning-Based Dynamic Framed Slotted ALOHA for Collision Arbitration in Active RFID Systems," in Advances in Grid and Pervasive Computing, 2007, pp. $655-665$.

[5] Z. Wang, D. Liu, X. Zhou, X. Tan, J. Wang, and H. Min, "Anti-collision Scheme Analysis of RFID System," Auto-ID Labs White Paper, 2007, http://www.autoidlabs.org/single-view/dir/article/6/281/page.html.

[6] F. C. Schoute, "Dynamic Frame Length ALOHA," IEEE Transactions on Communications, vol. 31, no. 4, pp. 565-568, 1983.

[7] J. R. Cha and J. H. Kim, "Novel Anti-collision Algorithms for Fast Object Identification in RFID System," in ICPADS '05: Proceedings of the 11th International Conference on Parallel and Distributed Systems - Workshops. Washington, DC, USA: IEEE Computer Society, 2005, pp. 63-67.

[8] W. T. Chen, "An Efficient Anti-Collision Method for Tag Identification in a RFID System," IEICE Transactions, vol. 89-B, no. 12, pp. 3386$3392,2006$.

[9] H. Vogt, "Efficient Object Identification with Passive RFID Tags," in Pervasive '02: Proceedings of the First International Conference on Pervasive Computing. $\quad$ London, UK: Springer-Verlag, 2002, pp. 98113.

[10] C. Floerkemeier, "Bayesian Trnasmission Strategy for Framed ALOHA Based RFID Protocols," in RFID, 2007. IEEE International Conference on RFID Gaylord Texan Resort, Grapevine, TX, USA, 2007, pp. 228235 . 